\title{
Reliability Prediction Of Constant Fraction Discriminator Using Modified PoF Approach
}

\author{
Adithya Thaduri, Lulea University of Technology \\ A K Verma, PhD, Stord/Haugesund University College \\ V Gopika, PhD, Bhabha Atomic Research Centre \\ Uday Kumar, PhD, Lulea University of Technology
}

Keywords: Accelerated Testing, Constant Fraction Discriminator, Design of Experiments, Physics of Failure, Radiation testing.

\section{SUMMARY AND CONCLUSION}

In this paper, the introduction, functioning and importance of constant fraction discriminator in nuclear field was studied. Furthermore, reliability and degradation mechanisms that affects the performance of output pulse with temperature and dose rates acts as input characteristics was properly explained and verified with the experiments. Accelerated testing was carried out to define the life testing of the component with respect to degradation in output TTL pulse amplitude. Time to failure was to be properly quantified and modelled accordingly.

\section{INTRODUCTION}

The reliability of electronic systems, used in nuclear power plants, was traditionally estimated with empirical databases such as MIL-HDBK-217, PRISM etc. These methods assign a constant failure rate to electronic devices, during their useful life. The constant failure rate assumption stems from treating failures as random events. Currently, electronic reliability prediction was moving towards applying the Physics of Failure (PoF) approach which considers information on process, technology, fabrication techniques, materials used, etc. Even alone PoF does not provide the reliability of the device. So amalgamation of deterministic and probabilistic analysis was carried out.

Electronics division of Bhabha Atomic Research Centre (BARC) was engaged in design \& fabrication of Complementary Metal Oxide Semiconductor (CMOS) and Bipolar Junction Transistor (BJT) Application Specific Integrated Circuits (ASIC) for nuclear pulse processing. These new microelectronic devices often exhibit infant mortality and wear-out phenomena while in operation. It points to competing degradation mechanisms-electro migration, hot carrier injection, dielectric breakdown etc.-that makes a device's useful life different from that predicted by empirical methods. Understanding the dominant mechanisms that lead to device failure PoF was a more realistic approach to reliability prediction. This report describes common failure mechanismsencountered in CMOS and BJT ICs and the efforts being taken to quantify these effects for several devices.
Neutron Flux Monitoring System (NFMS) comprises of different modules (Pulse Translator, Logarithmic Count Rate, Mean-Square Value Processor etc) that process pulse and current signals from detector. Besides, there are modules that generate trip signals. Trip signals are of $24 \mathrm{~V}$ level and optically isolated. It was worthwhile to study the failure mechanisms of the components involved in the signal processing chain of NFMS, as its reliability was being evaluated with conventional MIL-HDBK-217 method. The physics of failure study of these components will generate reliability data that can be eventually compared with the MTBF figures provided by MIL-HDBK-217. A few components have been identified in this regard; they form a part of trip signal generation which has direct implication on safety, Optocoupler, Instrumentation Amplifier, operational amplifier etc., are widely used in the trip modules of NFMS. So it was beneficial to start the PoF study with these components.

Another candidate chosen for study was Constant fraction discriminator (CFD-2004). This chip was used in the pulse processing circuits of NFMS. It was an indigenously developed ASIC which uses BJT process technology. CFD was another device which was failing regularly in the field and made up of BJT technology. It was a level discriminator at which it provides a pulse when the analog input reaches particular voltage level. A modified methodology was implemented which combines the physics of failure approach and statistical methods. The possible failure mechanisms for this device was electro migration and hot carrier injection. Temperature and radiation considered as stress parameters. These parameters effects the operation of BJT transistors inside to behave as it reduces the output voltage which further reduces the performance parameter which was in this case was voltage of output pulse. Constraint in the experimentation was that both the stress parameters cannot be applied simultaneously instead one after another. Effect of individual parameter was quantified on voltage output and degradation of whole device was studied. Selection of stress levels can be carried out by using Design of Experiments (DOE) and accelerating testing was implemented. Since there was no sufficient model for this device, modelling was to be carried 
out by probabilistic analysis. From the model, relation between design parameters and time to failure was observed and provided to designer.

\section{CONSTANT FRACTION DISCRIMINATOR}

Discriminators generate logic pulses in response to input signals exceeding a particular threshold. In general, there are two main types of discriminators, the leading edge discriminator and the constant fraction discriminator. The leading edge discriminator was the simpler one. Given an input pulse, the leading edge discriminator produces an output pulse at the time when the input pulse crosses a given threshold voltage. This, however, causes a problem in situations where the timing was important. If the amplitude was changed, but the rise time of the input pulse remains the same, a sort of "time walk" occurs [1]. That is, an input pulse with smaller amplitude but with the same rise time as a larger pulse will cross the threshold at a later time. Thus, the timing of the output pulse was shifted by this change in amplitude.

The constant fraction discriminator alleviates this problem by using a constant fraction, $f$, of the input pulse to precisely determine the timing of the output pulse relative to the input signal. It does this by splitting the input signal: attenuating one half so that it was a certain fraction, $f$, of the original amplitude, and delaying and inverting the other half. The attenuated pulse and the delayed and inverted pulse are then added together, and the zero crossing was computed.

The zero crossing gives the time at which the CFD should create an output pulse, and was always independent of amplitude. For a simple linear ramp, like the one shown above, the equations for its input pulse, attenuated pulse, and delayed and inverted pulse are as follows:

Input pulse, $V i=-A t$, attenuated pulse $V a=-f A t$ and when added together generates delayed and inverted pulse $V d=A(t-$ $t d$ ) where $t d=$ delay, $f=$ fraction, $A=$ initial amplitude

To find the zero crossing, set $V a+V d=0$ and solve for $t$ :

$0=-f A t+A(t-t d) \Rightarrow t_{\text {cross }}=t d /(1-f)$

Ideally, the delay was chosen such that the maximum of the attenuated pulse crosses at the desired fraction of the delayed pulse. In that case, $t_{\text {d_ideal }}=t_{\text {rise }}(1-f)$. However, if the delay was chosen smaller than $t_{d \_ \text {ideal }}, \mathrm{CFD}$ will operate at a fraction less than that of $f$. From equation 1, we can see that $t_{\text {cross }}$ was independent of the amplitude of the input pulse. The CFD has a monitor output feature, which outputs the bipolar signal created by summing the attenuated and the delayed and inverted pulses, so that we can view how it was calculating the zero crossing [8].

\section{FAILURE PHENOMENON OF CFD}

Here we have taken Constant Fraction Discriminator CFD 2004 made by Bharat Electronics Limited (BEL) Bangalore, India. Comparators and flip flops as shown in Figure 6 consist of transistors made up of BJT Technology. So, the failure physics of these transistors at wafer level adversely affects the performance and failure of CFDs. As from the description, if the wave from the counter expects to cross at a threshold level, CFD must provide Transistor Transistor Logic (TTL) pulse. Failure possibly happen if the threshold level at the input and the pulse width at the output varies in accordance with the prescribed level with the existing internal parameters. By the physics of failure approach, the stress parameters affect the BJT transistors to change their behavior of electrical hparameters. Commonly, when an electrical or temperature stress applied on the transistor, they develops reverse current from emitter to base to increase in such a way to degrade the performance of output electrical characteristics such as collector current and $\mathrm{V}_{\mathrm{CE}}$ voltage at the output. If these values changes inside the device, as all other devices are interconnected, this effective voltages and currents tend to vary at the larger levels of the whole device and output pulse width and time periods change. If this change was such a large that it can't detect the input pulse providing the output TTL, then it was considered as failure.

\subsection{Effect of Temperature}

The temperature dependence of bipolar transistors depends on a multitude of parameters affecting the bipolar transistor characteristics in different ways. Important effect was the temperature dependence of the current gain. Since the current gain depends on both the emitter efficiency and base transport factor [3].

The base transport was more likely to be temperature dependent since it depends on the product of the diffusion constant and carrier lifetime. The diffusion constant in turn equals the product of the thermal voltage and the minority carrier mobility in the base. The recombination lifetime depends on the thermal velocity. The result was therefore moderately dependent on temperature. Typically the base transport reduces with temperature, primarily because the mobility and recombination lifetime are reduced with increasing temperature. Occasionally the transport factor initially increases with temperature, but then reduces again.

Temperature affects the AC and DC characteristics of transistors. The two aspects to this problem are environmental temperature variation and self-heating. Some applications, like military and automotive, require operation over an extended temperature range. Circuits in a benign environment are subject to self-heating, in particular high power circuits.

Leakage current $I C O$ and current gain $\beta$ increase with temperature. The DC $\beta h F E$ increases exponentially. The AC $\beta$ hfe increases, but not as rapidly. It doubles over the range of $-55^{\circ} \mathrm{C}$ to $85^{\circ} \mathrm{C}$. As temperature increases, the increase in hfe will yield a larger common-emitter output, which could be clipped in extreme cases. The increase in $h F E$ shifts the bias point, possibly clipping one peak. The shift in bias point was amplified in multi-stage direct-coupled amplifiers. The solution was some form of negative feedback to stabilize the bias point. This also stabilizes AC gain [4].

As from the studies from BJT technology, temperature and radiation was selected as stress parameters. The emitter and collector current of npn BJT was given as Equation (2) and (3).

$$
\begin{gathered}
I_{E}=I_{E S}\left(e^{\frac{V B E}{V T}}-1\right) \\
I_{C}=\alpha_{T} I_{E S}\left(e^{\frac{V B E}{V T}}-1\right)
\end{gathered}
$$

The output voltage VCE was given as in Equation (4)

$$
V_{C E}=V_{C E}-I_{C} R_{e f f}
$$


where $R_{\text {eff }}$ was effective output resistance at the output, $I_{E S}=$ reverse saturation current at base-emitter diode, $\alpha_{T}=$ common base forward short circuit gain, $V_{T}=$ Thermal Voltage kT/q, $V_{B E}=$ base-emitter Voltage, $V_{C E}=$ base-collector Voltage, $V_{C C}$ $=$ Source Voltage typically $5 \mathrm{~V} / 10 \mathrm{~V}$.

In Eber-Moll Model, IC grows at about $9 \% /{ }^{0} \mathrm{C}$ if you hold $V B E$ constant and $V B E$ decreases by $2.1 \mathrm{mV} /{ }^{0} \mathrm{C}$ if you hold IC constant with the temperature.

Since both the currents depend on temperature parameter $V_{T}$, the raise in the temperature leads to vary these parameters which finally lead to degrade the performance of CFD.

\subsection{Effect of Radiation}

Another stress parameter which degrades the BJT devices was $\beta$-radiation. Degradation of many types of bipolar transistors and circuits was known to depend strongly on dose rate. For a given total dose, degradation was more severe in low dose rate exposure than high dose rate exposure. This effect has been attributed to space charge effects from trapped holes and hydrogen related species through oxygen vacancies in base oxide. There are several hardness assurance tests and most popular has been high dose rate irradiation at elevated temperatures [5].

Although radiation exposure generally leads to grain degradation in npn and pnp devices, the mechanisms by which radiation effects their gains are quite different. Ionizing radiation degrades the current gain of npn bipolar transistors by introducing net trapped positive charge and interface traps into the oxide base. This positive oxide trapped charge spreads the emitter-base depletion region into the extrinsic base results in increase of base recombination current under forward-bias operation at the junction. Radiation-induced interface traps, especially those near mid-gap, serve as generationrecombination centers through which recombination current in the base was further increased due to enhanced surface recombination velocity. In pnp transistors [6], near-midgap interface traps in the base oxide also increase the base current by surface recombination. Compared with npn transistors, radiation-induced net positive oxide trapped charge can mitigate gain degradation by creating an imbalance in carrier concentrations at the surface of the base.

From the statistical results explained in et al. Witczak [5], Current gain degradation grows worse with decreasing dose rate regardless of dose. Excess base current, an increase in base current due to radiation exposure, increases gradually with decreasing dose rate. This effect was due to weak dependence of excess base current on radiation-induced defect densities at large total dose. Changes in collector current as compared to base current was small because it provides meaningful assessment of amount of gain degradation while relating closely to the physical mechanisms, excess base current was a convenient parameter to evaluate radiationinduced damage in these devices [7].

Although much progress has been made in understanding the effects of dose rate and temperature on radiation-induced bipolar gain degradation, still there was ambiguity in selecting the optimum values for assurance testing. From the analysis carried out by Witczak [5], the combined influence of both radiation and temperature has considerable dependence on gain degradation and excess base current enhancement. The combine effect of temperature and radiation results in degradation of performance parameters such as threshold voltage, pulse amplitude and time period of Constant Fraction Discriminator. The radiation exposure was exposed to CFD for 0, 3,6.5 and 10 Kgray respectively.

\section{EXPERIMENTAL SETUP}

CFD 2004 made up of BJT technology manufactured from BEL was considered for this study. It was 24-pin Dual in Package (DIP) plastic package with operating conditions $5.2 \mathrm{~V}$ to $5 \mathrm{~V}$ and operating temperature $100^{\circ} \mathrm{C}$ and circuit for testing was shown in figure 1.

In order to monitor and test this IC for temperature and radiation considering time, a circuit was required to assess and measure it. Figure 8 shows the conditional measuring circuit for this failure testing. As we can see, along with IC, several other components also required to measure the performance parameters such as Inverter (to invert the positive pulse to negative pulse input which was not shown in figure), resistors, capacitors, etc. These components are also sensitive to temperature which effects the measurement.

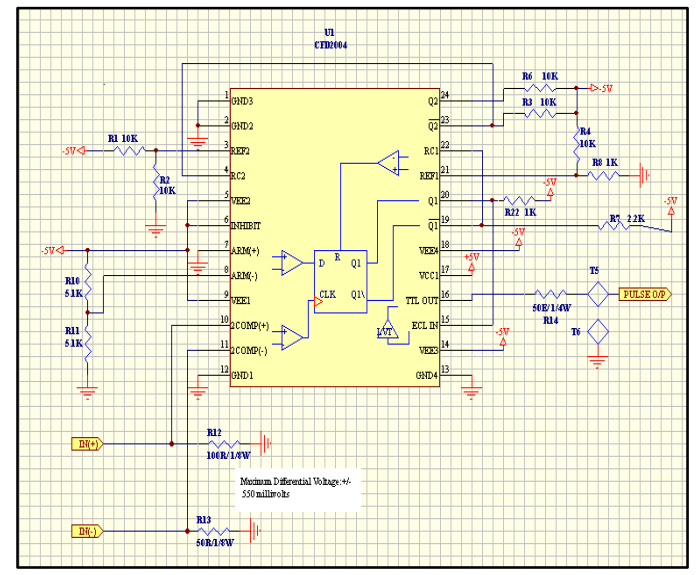

Figure 1: Circuit Diagram of CFD.

Hence, the circuit was divided to two parts: CFD part and measurement part. In this way, effect of temperature lies only on CFD. The board of CFD part was also holed out at the bottom of IC to expose it with sufficient temperature. A thermistor was placed across IC to continuously monitor the temperature of it.

\section{DESIGN OF EXPERIMENTS}

From the research, temperature and radiation dose rate are considered as stress parameters for the degradation of performance of constant fraction discriminator. The performance parameters include threshold voltage which was at the input and TTL pulse amplitude at the output. From the Taguchi method of design of experiments, selection of this temperature levels was considered such that IC provides optimum performance. Testing can be done in two steps: Basic and Extensive testing for detailed analysis of CFD. At each stage, temperature and radiation stress parameters were applied one after another. 


\subsection{Stage 1: Basic testing}

\subsubsection{Screen Testing:}

Radiation and temperature are the dominant stress parameters. Initially the device was exposed to radiation under biased conditions. Thereafter it was exposed to temperature. The selection parameters can be done using 2-stage DOE. Accelerated testing was carried out from the data acquired from DOE to define time to failure of device using Response Surface Method (RSM) method.

\subsubsection{Radiation Testing:}

In this study, variation of pulse amplitude and time period in accordance with the radiation dose rate was calculated. With sample size of 3 with radiation levels from 20 mins to 40 mins, a small variation in the output voltage was observed.

\subsubsection{Temperature Testing:}

In this study, variation of pulse amplitude and time period in accordance with the temperature was calculated. Range of temperature was considered from $30^{\circ} \mathrm{C}$ to $50^{\circ} \mathrm{C}$. The results showed considerable decrease in the amplitude as the temperature increased nonlinearly to $50^{\circ} \mathrm{C}$. As from these results, temperature variation on CFD decreases time period linearly. This result depicts some change in performance parameters with temperature.

From the screening results, we get to know that both radiation and temperature degrades the performance parameters as both the parameters increases. This experiment validates the on degradation mechanism that was earlier discussed.

\subsubsection{Design of Experiments:}

In this stage, stress levels of both radiation and temperature was subjected to CFD circuit one after another as with radiation dosage of 20 mins to 40 mins and temperature range from $30^{\circ} \mathrm{C}$ to $50^{\circ} \mathrm{C}$ using $2 \times 2$ matrix. From the design of experiments and the response surface analysis, the levels of input parameters was selected such a way that maximum degradation of performance parameter was expected and acts as input to the accelerated testing of CFD for life testing analysis.

\subsubsection{Accelerated Testing:}

From the inputs of DOE, the levels are selected such that pulse amplitude as maximum degradation. The values are provided below and experiments are carried out at regular intervals after exposure of radiation and maintaining the temperature level with extended period of time upto 200 hours. The degradation of pulse amplitude happened over the time non-linearly from the results. To quantify and model this time to failure considering dose rate and temperature need to be studied as there are no physics of failure models available in the literature.

\subsection{Stage 2: Extensive testing}

From the input from the stage 1, as the both temperature and radiation parameters increases, the output performance factor further degrade. In this stage, the radiation parameters are selected at the higher dosages as 0 KGray, 3.14 KGray, 6.64 KGray and 10 KGray. Similarly, the testing of IC was excited to higher temperatures of $30^{\circ} \mathrm{C}, 50^{\circ} \mathrm{C}, 70^{\circ} \mathrm{C}$ and $90^{\circ} \mathrm{C}$. From the Witczak [5], the degradation of the device by radiation increases further with the temperature. Hence the items are subjected at first to the radiation step and second to the temperature step. To get more extensive data, accelerated testing was carried out after the temperature step. This radiation-temperature-time sequence was carried out at all the stress levels of the radiation. The temperature further degrades the effect of radiation step and shown in Figure 2.

Similarly, the degradation characteristics are observed with accelerated testing carried out $90^{\circ} \mathrm{C}$ at each radiation step and results are provided in Figure 3. The output parameter further degrades by the effect of accelerated time along with rise in radiation and temperature. From the above figures, it was concluded that both radiation and temperature degrades the performance parameter, the voltage of the output pulse with further accelerated time.

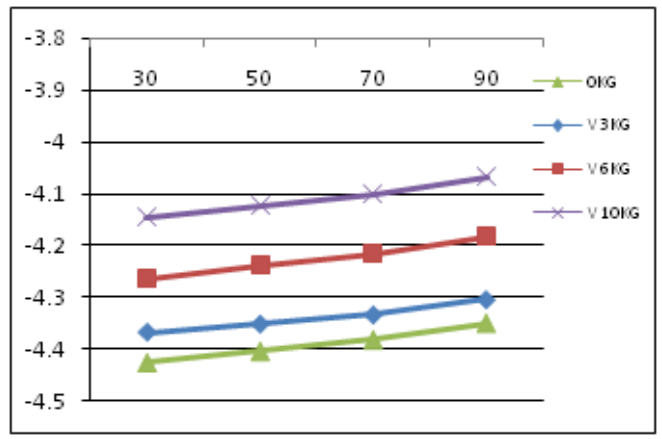

Figure 2: Degradation with radiation and temperature

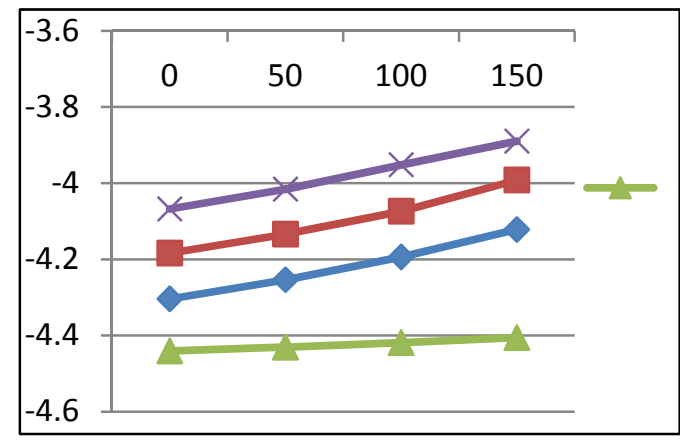

Figure 3: Degradation with radiation and accelerated time

\section{ANALYSIS OF RESULTS}

From the results obtained from the both the stages, the analysis of results was carried out to find the behaviour of individual parameters on the output performance parameter. In order to analyse and model the parameters, some tools such as Minitab and ALTA are used to characterize and model the output voltage parameter. The 3D scatter plot with radiation, temperature and accelerated time as axis parameters and output voltage as the points in Figure 4. As discussed in the above section, the voltage degrades with increase in radiation, temperature and accelerated time. The data points represent 
the data acquired from both design of experiments and accelerating results.

\section{Characterization of Stress parameters:}

The characterization of each input stress parameter with the degraded output voltage was carried out using contour plots and matrix plots are shown in Figure 5. By observing the plots, the degraded parameter was characterized by each stress parameter and behaves direct proportional to the degradation of output voltage pulse due to the fact that all these parameters induce defects into the device that reduces the response of the item.

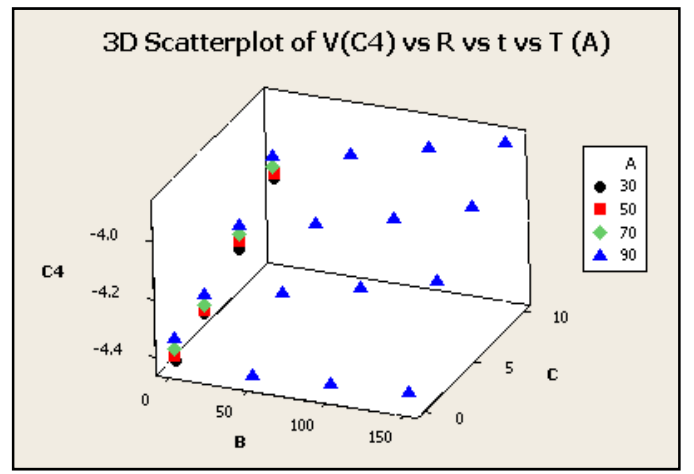

Figure 4: 3D scatter plot of Radiation, Temperature and accelerated time with output voltage

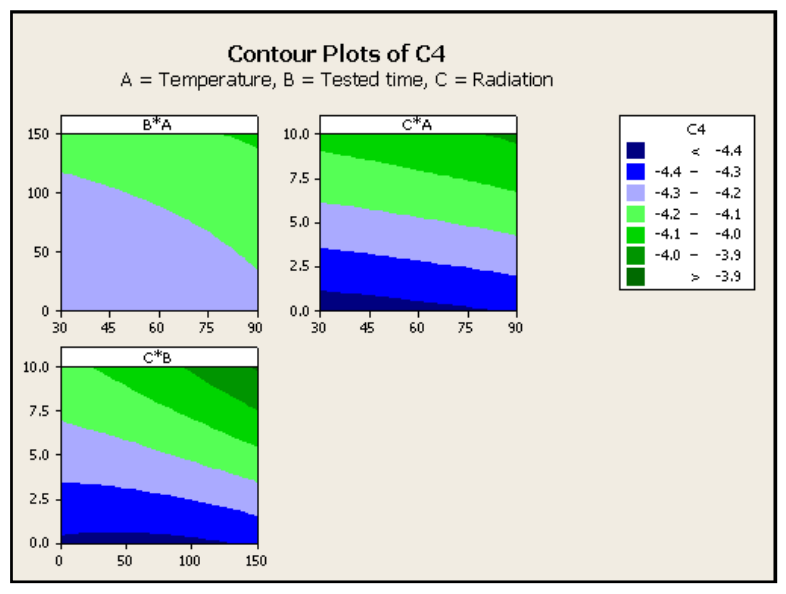

Figure 5: Contour plots of stress parameters

Each stress parameter was solely fitted as linear plots with the output parameter along with ANOVA and regression life data.

\subsection{Fitted Line plots:}

The fitted linear plot provides the linearity behaviour of the one stress parameter at a time on the output parameter. The fitted line plot provides not only the estimated regression function, but also a scatter plot of the data adorned with the estimated regression function. The linear plots of temperature, radiation and time stress parameters with equations were provided in Eqns 5, 6 and 7.

$$
\begin{aligned}
& V=-4.385+0.002278 T \\
& V=-4.266+0.001086 t \\
& V=-4.393+0.03570 R
\end{aligned}
$$

\subsection{One-Way ANOVA}

One-way ANOVA was a technique used to compare means of two or more samples. It tests the null hypothesis that samples in two or more groups are drawn from the same population. If the group means are drawn from the same population, the variance between the group means should be lower than the variance of the samples, following the Central Limit Theorem. A higher ratio therefore defines that samples are drawn from different samples. One-way ANOVA of temperature, radiation and time stress parameters with the output voltage was shown in tables 5,6 and 7 .

Table 5: One-way ANOVA of temperature parameter

\begin{tabular}{|l|l|l|l|l|l|}
\hline Source & DF & SS & MS & F & P \\
\hline A & 3 & 0.0795 & 0.0265 & 1.08 & 0.376 \\
\hline Error & 24 & 0.5880 & 0.0245 & & \\
\hline Total & 27 & 0.6676 & & & \\
\hline
\end{tabular}

$\mathrm{S}=0.1565 \quad \mathrm{R}-\mathrm{Sq}=11.91 \% \quad \mathrm{R}-\mathrm{Sq}(\operatorname{adj})=0.90 \%$ Pooled StDev $=0.1565$

Table 6: One-way ANOVA of radiation parameter

\begin{tabular}{|l|l|l|l|l|l|}
\hline Source & DF & SS & MS & F & P \\
\hline A & 3 & 0.50312 & 0.16771 & 24.48 & 0.0 \\
\hline Error & 24 & 0.16444 & 0.00685 & & \\
\hline Total & 27 & 0.66756 & & & \\
\hline
\end{tabular}

$\mathrm{S}=0.08277 \quad \mathrm{R}-\mathrm{Sq}=75.37 \% \quad \mathrm{R}-\mathrm{Sq}(\mathrm{adj})=72.29 \%$

Pooled StDev $=0.0828$

Table 7: One-way ANOVA of temperature parameter

\begin{tabular}{|l|l|l|l|l|l|}
\hline Source & DF & SS & MS & F & P \\
\hline A & 3 & 0.1045 & 0.0348 & 1.49 & 0.244 \\
\hline Error & 24 & 0.5630 & 0.0235 & & \\
\hline Total & 27 & 0.6676 & & & \\
\hline
\end{tabular}

$\mathrm{S}=0.1532 \quad \mathrm{R}-\mathrm{Sq}=15.66 \% \quad \mathrm{R}-\mathrm{Sq}(\operatorname{adj})=5.11 \%$

Pooled StDev $=0.1532$

Since $\mathrm{P}<0.5$, null hypothesis was rejected for estimation.

\subsection{Individual Value plots}

Minitab's new individual value plot allows you to view important data features, to find miscoded values, and to identify unusual cases. An individual value plot can help you to set the appropriate course for your analysis and to avoid wasted time and frustration. Individual value plots to identify possible outliers and other values of interest. It can also clearly illustrate characteristics of the data distribution. Here, the mean value at each stress level of the factor was plotted across the output voltage to assess its performance degradation over in increase of the stress value.

\subsection{Linear Regression Analysis}

The linear regression equation with temperature, radiation and accelerated time was modeled as in equation

$V=-4.51+0.00106 T+0.000840 t+0.0357 R$ 
The positive sign of each parameter represents the output voltage degrades over increase of these parameters.

\subsection{Response Surface Regression:}

Response surface regression was the popular technique which provides estimates for individual factors and also its interaction effects on the output performance parameter. Each of the estimates (coefficients, indicated with Coeff) has a standard error - this was a measure of how variable the estimate was likely to be. To gain the $95 \%$ confidence intervals of the coefficient, we multiply the standard error by 1.96, and add and subtract this from the coefficient. The standard error of a coefficient (SE Coeff) was the square root of the corresponding diagonal element of the covariance matrix of the coefficient estimates. The variances are the diagonal elements of the $\mathrm{X}^{\prime} \mathrm{X}$ inverse matrix times the mean square error (MSE). T-value (T) computed from the data for testing the hypothesis that the corresponding population coefficient was 0 . The $p$-values for the test that the population value was 0 are given in the $\mathrm{P}$ column. Large t-values go with small $\mathrm{p}$-values and suggest a term contributes to the model. $T$ isn't very useful on its own, but it does give us $\mathrm{P}$ - that was the probability of the result occurring, if the real value in the population was zero.

Table 8: Coefficients of response surface regression

\begin{tabular}{|l|r|r|r|r|}
\hline Term & \multicolumn{1}{|c|}{ Coef } & \multicolumn{1}{l|}{ SE Coef } & \multicolumn{1}{c|}{ T } & P \\
\hline Constant & -4.21357 & 0.02718 & -155.003 & 0 \\
\hline A & 0.03642 & 0.01104 & 3.298 & 0.004 \\
\hline B & 0.06464 & 0.01104 & 5.853 & 0 \\
\hline C & 0.19755 & 0.01342 & 14.718 & 0 \\
\hline A*A & 0.00499 & 0.01861 & 0.268 & 0.791 \\
\hline B*B & 0.02193 & 0.01861 & 1.178 & 0.253 \\
\hline C*C & -0.01665 & 0.01436 & -1.16 & 0.261 \\
\hline A*C & 0.00696 & 0.01375 & 0.506 & 0.618 \\
\hline B*C & 0.05105 & 0.01375 & 3.714 & 0.001 \\
\hline
\end{tabular}

\section{$\mathrm{S}=0.0332874$ PRESS $=0.0607202$}

$\mathrm{R}-\mathrm{Sq}=96.85 \% \mathrm{R}-\mathrm{Sq}$ (pred) $=90.90 \% \mathrm{R}-\mathrm{Sq}($ adj $)=95.52 \%$

The above results show stability and how well was the prediction of parameters as in Table 8 . S was the square-root of mean square error and PRESS statistic in the original units of the response when a power transformation of the response was applied in a linear regression. R-Sq evaluates how closely the data fall next to the fitted line. Here prediction R-Sq was $90.9 \%$ because of the missing null values exists in time parameter. So R-Sq was adjusted to $95.52 \%$ which was a quite reasonable prediction for the degradation of output variable.

The analyais of variance of these coefficients were produced in Table 9. DF refers to the degrees of freedom for each source. The SS column gives, top to bottom, the sums of squares SSR, SSE and SST. The SSE was used (with the formula and a calculator) for the F-test for testing some subset of the independent variables. Here P-value was also significant predictor as how well the term contributes to the overall output parameter. Interaction has significant value which concludes the interaction of temperature and radiation has essential effect on degradation of output voltage.

Table 9: Analysis of Variance

\begin{tabular}{|c|c|c|c|c|c|c|}
\hline Source & DF & Seq SS & Adj SS & Adj MS & F & P \\
\hline Regression & 8 & 0.64651 & 0.64651 & 0.080814 & 72.93 & 0 \\
\hline Linear & 3 & 0.616349 & 0.327607 & 0.109202 & 98.55 & 0 \\
\hline Square & 3 & 0.00303 & 0.00303 & 0.00101 & 0.91 & 0.454 \\
\hline Interaction & 2 & 0.027131 & 0.027131 & 0.013566 & 12.24 & 0 \\
\hline $\begin{array}{c}\text { Residual } \\
\text { Error }\end{array}$ & 19 & 0.021053 & 0.021053 & 0.001108 & & \\
\hline Total & 27 & 0.667563 & & & & \\
\hline
\end{tabular}

The final part of the output was some diagnostics, to help you to interpret the equation. The Minitab tool has selected some cases it believes you might want to look and bases this on the residuals and the influence. The eestimated Regression Coefficients for $\mathrm{V}$ using data in uncoded units was modeled as

Vout $=-4.4583+0.00032 T-4.035 t+0.0332 R+$ $5.546 \times 10^{-6} T^{2}+3.898 \times 10^{-6} t^{2}-6.6597 \times 10^{-4} R^{2}+$ $4.639 \times 10^{-5} T R+0.00014 t R$

The failure criteria were measured as $5 \%$ degradation from the initial value and by considering the normal operation conditions, the time to failure was calculated as $5.7 \times 10^{7} \mathrm{hrs}$. This more accurate figure decreases in the replacements costs thus by ensuring efficient usage of the device.

\section{ACKNOWLEDGMENT}

The authors are indebted to Bharat Electronics Limited, for manufacturing and extensive datasheet of the CFD samples. The authors acknowledge Rajesh Gopinath for his extensive inputs in reliability testing of electronic samples and ISOMED, BARC for their support in radiation testing of ICs. In addition, authors thank Prof. V Ramgopal Rao, IIT Bombay for his knowledge and support for information of technology perspective on these devices.

\section{REFERENCES}

1. "Failure of the Constant Fraction Discriminator", Kristin Luery, July 9, 2003.

2. "VERITAS L1 trigger Constant Fraction Discriminator", Vladimir Vassiliev, Jeremy Smith, David Kieda.

3. "Principles of Electronic Devices", B. Van Zeghbroeck, 2011.

4. "The temperature characteristics of bipolar transistors fabricated in CMOS technology", Guijie Wang, Gerard C.M. Meijer, Elsevier, Sensors and Actuators 872000 81-89.

5. "Hardness assurance testing of bipolar junction transistors at elevated irradiation temperatures", S.C. Witczak, R.D. Schrimpf, D.M. Fleetwood, K. F. Galloway, R.C.Lacoe, 
D.C. Mayer, J.M. Puhl, R.L.Pease, J.S. Suehle, IEEE transaction on Nuclear Science, Vol 44, No. 6, Dec 1997

6. "Charge separation for bipolar transistors", S.C. Witczak, R.D. Schrimpf, D.M. Fleetwood, K. F. Galloway, R.C.Lacoe, D.C. Mayer, J.M. Puhl, R.L.Pease, J.S. Suehle, IEEE transaction on Nuclear Science, Vol 40, pp 12761285,1993
7. "Modelling ionizing radiation induced gain degradation of the lateral pnp bipolar junction transistor", R.D. Schrimpf, D.M. Schmidt, D.M. Fleetwood, R.L.Pease, W.E. Combs, IEEE transaction on Nuclear Science, Vol 43, pp 30323039, 1996

8. "Constant fraction Discriminators", Kevin Carnes, Jan 2003 